\title{
El uso pragmático del diminutivo en el español del Valle Central de Costa Rica
}

\author{
Elber Aguilar Porras \\ Escuela de Literatura y Ciencias del Lenguaje \\ Universidad Nacional, Costa Rica
}

\begin{abstract}
Resumen
En este artículo se realiza un análisis de los diminutivos más frecuentes utilizados en el español del Valle Central de Costa Rica. Desde una perspectiva pragmática, y haciendo uso de diversos programas computacionales para el análisis de la frecuencia de los diminutivos, se ofrece una visión más detallada del uso real que los interlocutores les dan. En este estudio, se toman en cuenta los usuarios de la red social Facebook y se considera, asimismo, el rol que cumplen los diminutivos en los diversos actos de habla y cómo estos afectan positiva o negativamente la imagen de los interlocutores.
\end{abstract}

Palabras claves: Antconc, Atlas-Ti, cortesía, atenuador discursivo, imagen positiva, imagen negativa, actos locutivos, actos ilocutivos y actos perlocutivos, actos fallidos

\begin{abstract}
In this article, an analysis of the most frequent diminutives used by native speakers of Spanish from the Central Valley of Costa Rica is presented. From both a pragmatic perspective and the application of specialized software designed for the frequency of words, this research presents a detailed and broader understanding of the real use of diminutives. In this research paper, Facebook users are taken into consideration as well as the role diminutives play in different speech acts and how these positively or negatively affect the participants' face.
\end{abstract}

Key words: Antconc, Atlas-Ti, politeness, discourse attenuator, positive face, negative face, illocutionary and perlocutionary acts, failed speech 


\section{Introducción}

$\mathrm{U}$ n diminutivo es considerado, comúnmente, como no más que un sufijo, el cual es agregado a una palabra o raíz para disminuir su valor léxico. Sin embargo, el uso de un sufijo para crear una entrada léxica diminutiva supone, en el caso del español, solamente una de sus estrategias para formar palabras.

Los diminutivos, asimismo, cumplen también diversas funciones pragmáticas y se utilizan para expresar diversos significados, todos en su mayoría dependientes de su contexto discursivo inmediato, por lo que se debe de tomar una posición más pragmática que sintáctica, incluida la morfología, para entender su función social. Por tal razón, generalizar el término diminutivo para referirse a todas las posibles funciones pragmáticas que este desempeña en el lenguaje del español es demasiado arriesgado desde el punto de vista de la lingüística.

\section{La pragmática}

La pragmática se define como el estudio de los principios que regulan el uso del lenguaje en la comunicación, es decir, las condiciones que determinan tanto el empleo de un enunciado concreto por parte de un hablante específico en una situación comunicativa dada, como su interpretación por parte del destinatario. Esta disciplina toma en consideración factores extralingüísticos que determinan el uso del lenguaje (factores a los que no puede hacer referencia un estudio gramatical). Así, se da una interpretación de la competencia comunicativa más que una in- terpretación literal. Considerando la sintaxis, recordemos que ésta se ocupa más del análisis de la relación existente entre los diferentes símbolos o signos del lenguaje. Se trata de una visión que se interesa por el orden de los constituyentes, los cuales en algunas lenguas son más fijos y en otras este orden es más flexible (Vidal, 1996, p. 170-174). El orden de elementos o signos podría causar un cambio en los significados, lo cual sería algo meramente relacionado con la pragmática. Esto, podríamos decir, sería una relación o similitud que la sintaxis comparte con la pragmática. Recordemos que el orden sintáctico de los elementos que causan diferencias de significados, para que se convierta en pragmático, debería ser cuando la idea no es clara por sí sola, dependiendo de elementos contextuales.

\section{El corpus}

El corpus seleccionado para llevar a cabo esta investigación se basó en conversaciones tomadas de Facebook. Esta red social es una de las más populares del mundo, la cual inició como un proyecto local y se ha expandido por todos los continentes. Facebook comenzó como un proyecto aislado por parte de un estudiante de la Universidad de Harvard, Mark Zuckerberg, quien pretendía crear una forma sencilla de comunicación entre los alumnos de esta universidad. No obstante, dicha idea dio pie a que poco a poco cientos de usuarios se sumaran, debido a que sus fundadores decidieron abrir la cuenta a usuarios a los cuales solo se les pedía como requisito tener un correo electrónico. Para 2007, Facebook ya era traducido al español, alemán y francés, como 
lo señaló el diario El País (2008). Según el diario argentino La Nación (2014), para 2013, Facebook contaba con 1230 millones de cuentas. Desde sus inicios, alrededor del año 2004, y hasta hoy, Facebook tiene más de 1280 millones de usuarios, de acuerdo con el diario español Cinco Días (2014).

Facebook ofrece, entre las más conocidas y usadas, una lista de servicios que se deben tener claros. La "lista de amigos" de Facebook es uno de los servicios más conocidos de la aplicación. En este servicio, el usuario decide si acepta o envía una invitación a una persona que quizás conozca. Otro es el "chat", donde los usuarios pueden enviar mensajes instantáneos. También existen los "grupos y páginas" donde se puede reunir a personas con intereses comunes; este tipo de servicios no ofrece interacción. El "muro" es un espacio donde las demás personas hacen publicaciones (fotos, videos o simplemente texto) con el fin de que el usuario (registrado) las pueda ver. Entre otros servicios, se puede apuntar el botón de "like" (me gusta), las "aplicaciones", "juegos" y, recientemente (2011), la "biografía", que viene a sustituir el "muro" y tiene como objetivo agilizar y optimizar el paseo de los usuarios por los perfiles de todos los contactos; se puede ver en esta la fecha exacta de publicaciones, actualizaciones de estado y comentarios. En la metodología, se mencionarán y justificarán los servicios de Facebook que se tomarán en cuenta para este estudio.

Una de las características principales de Facebook es su carácter público y su mezcla discursiva oral y escrita, lo cual aumenta el valor de los datos de esta investigación. A pesar de que inició como una red interpersonal de comunicación, Facebook, al igual que sus homólogas, es un sistema de comunicación abierto al público; dicha característica es lo que permite que este tipo de redes crezca cada vez más. De hecho, en la política de privacidad de la red social se aclara que "Eres [el usuario] el propietario de todo el contenido y la información que publicas en Facebook, y puedes controlar cómo se comparte a través de la configuración de la privacidad y de las aplicaciones ${ }^{1 "}$. De tal forma, al no asignarle restricción a ninguna publicación, la persona deja de tener derechos sobre esta, puesto que, como se apunta en la política de privacidad, existen herramientas para evitar que lo que se publica sea tomado por terceros. Esta aclaración es muy relevante por cuanto es esta red social la base para el estudio que se lleva a cabo, así como por razones legales.

Por otro lado, cabe añadir que otra característica de Facebook es el carácter oral-escrito de su discurso. Se puede pensar que es solamente un discurso escrito debido a que por este medio se plasman las ideas de los usuarios. Sin embargo, se puede notar cómo abundan las características propiamente orales del idioma (español en este caso), como son las interjecciones u onomatopeyas, emoticones y, mayormente, las supuestas faltas de ortografía que, en muchos casos, son solamente la reproducción fonológica del idioma mismo. Por tal motivo, y a modo de ampliar la justificación de esta investigación, se ha seleccionado dicha red social como base del presente estudio puesto que, en primera instancia, es una forma efectiva de recolectar datos sin restricciones. Esta característica discursiva ayudará a eliminar los efectos de influencia del investigador en las investigaciones. 
Así mismo, por tener rasgos tanto escritos como orales, es una manera más íntegra de analizar datos y, por tanto, de obtener resultados más fidedignos y fiables.

\section{Justificación de la investigación}

Para poder realizar un análisis significativo del uso real que se le da a los diminutivos por parte de los hablantes del español de Costa Rica, específicamente de la zona del Valle Central, se debe seleccionar cuidadosamente el corpus de donde se obtendrán los datos en que los informantes utilicen un lenguaje natural, sin sentirse monitoreados y sin que ello implique una modificación de su habla, utilizando quizás una jerga distinta de la usada normalmente.

Cuanto más alta la relación familiar o cercanía con la persona, mayores serán las probabilidades del uso de los diminutivos. Esto justifica por qué no se seleccionaron fuentes más formales como noticieros, revistas o bien periódicos, ya que la gran mayoría de estas fuentes incluyen un lenguaje más formal que el que se da en conversaciones más familiares. Esta característica discursiva se puede encontrar en Facebook, lo cual ayudará a eliminar los efectos de influencia del investigador en las investigaciones.

Es importante resaltar que los diminutivos no siempre son designados para disminuir el valor de una entrada léxica y, en algunas ocasiones, se relacionan más bien con formas peyorativas del lenguaje en sí. Términos como qué peliculita designan nada más que una expresión negativa, donde se resalta por medio del diminutivo dicha forma peyorativa. Spitzer (p.201-202) da especial énfasis a este componente lúdico en el empleo de los sufijos diminutivos. Igualmente, Borja (2010) hace referencia a esta función negativa del diminutivo. Según él, "Esta marcación se usa sobre entidades que causan una tensión emocional negativa [opuesta a la positiva que según él causa una tensión emocional positiva sobre el hablante/conceptualizador en términos de aprecio o afición] sobre el hablante/ conceptualizador en términos de desprecio o menosprecio". Por medio de los diminutivos, entonces, el lenguaje adquiere una función más amplia, al salirse de lo normal y esperado, pasando así a formas irónicas. En la ironía, para Borja (2010), "el hablante/ conceptualizador parece incrementar el choque con una realidad desagradable y con ello logra una marcada jerarquización de valores al interior de la escena discursiva." Así, por razones obvias, nos debemos preocupar por el contexto si se quiere entender mejor el uso de estos diminutivos. Es por esta multifuncionalidad de los diminutivos, y por la poca consideración contextual que se le ha dado en diferentes estudios, que se deben explorar sus usos en contextos reales y, de esta forma, concientizar sobre el uso real que implica un diminutivo, ampliando de este modo la exclusividad de disminución del valor léxico que, equívocamente, se le ha atribuido.

\section{Consideraciones conceptuales}

Desde la pragmática, se deben de considerar los siguientes actos de habla para atender la correcta interpretación de los enunciados: los actos de habla locutivos, ilocutivos y perlocutivos así 
como los actos fallidos. Podemos apuntar que los primeros son las ideas o conceptos de las frases, aquello que se dice ordenado en morfemas y palabras. Por su parte, un acto ilocutivo es la intención o finalidad concreta del acto de habla locutivo. Así entonces, al referirnos a los diminutivos podemos utilizar más bien el concepto de fuerza ilocutiva del diminutivo y analizarlo como tal. Los actos perlocutivos son los efectos o consecuencias, buscados o no buscados, que el enunciado (o un acto ilocutivo) produce en el interlocutor en una determinada circunstancia; dichos efectos pueden ser convencionales o no convencionales (Davis, 1980).

Los actos ilocutivos no necesariamente conllevan a un acto perlocutivo o, bien, puede que no produzcan el acto deseado. Interesa, entonces, analizar la fuerza perlocutiva de diminutivos con intenciones de atenuar (acto ilocutivo) en los enunciados, si las funciones comunicativas (acto ilocutivo) causan las (re)acciones deseadas en los hablantes (acto perlocutivo) o si simplemente conforman un acto fallido. Este último se define como las acciones defectuosas debido a la interferencia de algún deseo, conflicto o cadena de pensamientos inconscientes; son actos de habla que expresan lo contrario de lo que el hablante quería decir sin proponérselo.

Desde la perspectiva de Searle, los actos descritos anteriormente pueden ser categorizados como directos (son aquellos enunciados en los que el acto locutivo e ilocutivo coinciden, es decir, explicita la intención), o indirectos (son aquellas frases en las que las fuerzas locutiva e ilocutiva no coinciden, por lo tanto, la finalidad de la oración es distinta de lo que se expresa directamente). Searle se centra en la relación entre la intención del hablante y la interpretación del oyente.

En resumen, los actos de habla directos son los que están marcados por una correlación entre forma lingüística y fuerza ilocutiva. Si a una locución específica puede atribuírsele más de un objeto ilocutivo, esta sería un acto de habla indirecto. En nuestro objeto de estudio, no siempre se da un uso de los diminutivos con intenciones. Por otro lado, los diminutivos cumplen funciones atenuadoras por medio de las cuales se mitigan actos de habla, tales como las exhortaciones o bien la aserción (argumentación). Esta última se describe como "la intención del hablante de convencer al oyente de que él, es decir, el hablante, cree sinceramente que la proposición expresada corresponde a un estado de cosas real" (Haverkate, 1994, p.88-117). La imagen negativa, en términos de Brown y Levinson (ct. Haverkate, 1994, p.116-117), se define como el deseo de tener libertad de acción sin tener imposiciones; la imagen del receptor se ve afectada ante la intención del hablante de "convencer". El elemento atenuador del diminutivo mantiene ese balance social entre los hablantes como una manera de reparar daños en las imágenes, por tanto, constituye una estrategia del español.

Además de los actos de habla descritos anteriormente, se debe también considerar aquellos que atentan contra la imagen de los interlocutores como lo son los Face-threatening acts (FTAs) así como otros conceptos de gran importancia para la interpretación de las interacciones de los participantes en un acto de habla. Desde la perspectiva de Brown y Levinson (1987), “...face-threatening acts are at times inevitable based on the terms 
of the conversation... A face threatening act is an act that inherently damages the face of the addressee or the speaker by acting in opposition to the wants and desires of the other". Según esta definición, un FTA no es más que actuar, verbalmente, en contra de lo que la otra persona desea. Asimismo, Brown y Levinson hablan del concepto de imagen (Face), el cual versa sobre la idea de que cada hablante tiene una imagen (positiva-negativa) que se ve afectada o beneficiada en los actos de habla. Imagen es la visión que la sociedad tiene de nosotros. Según dichos autores, imagen se refiere a "...the want of every member that his wants be desirable to at least some others executors." (Brown y Levinson, 1978, p. 62). Adicionalmente, también se tiene una "imagen positiva" o una contraparte "negativa"; así, "the positive consistent self-image or 'personality' (crucially including the desire that this self-image be appreciated and approved of) claimed by interactants"; la imagen negativa se define como "the want of every competent adult member that his actions be unimpeded by others...the basic claim to territories, personal preserves, rights to non-distraction--i.e. the freedom of action and freedom from imposition" (Brown y Levinson, 1987, p. 61). Dichos conceptos forman parte de un hiperónimo llamado cortesía (politness), el cual, al igual que sus hipónimos, se subdivide en cortesía positiva y negativa. El primer término hace referencia a "... being complimentary and gracious to the addressee", mientras que "Negative politeness is found in the various ways of mitigating an imposition..." (Brown y Levinson, 1987, p.56-68). Como se observa, aparte de las intenciones de los hablantes expresadas en los diferentes actos de habla, también se deben de tomar en cuenta aquellos aspectos determinados por la sociedad que, sin duda, son de vital importancia para la interpretación de lo comunicado.

Finalmente, los apelativos y el cumplido son formas de tratamiento ${ }^{2}$ que se marcan por medio de los diminutivos y que, al igual que los conceptos anteriores, juegan un papel de importancia en la interpretación de las interacciones. Los apelativos, que se asemejan a los honoríficos, se utilizan como vocativos para resaltar la diferencia honorífica. Estos hacen referencia a formas léxicas tales como adjetivos o sustantivos, utilizados en relaciones personales, y asociados al rango social y cuestiones de género. Con respecto al cumplido, el cual tiene como objeto "...crear o mantener un ambiente de amabilidad...[o bien] expresar solidaridad y aprecio...", supone una forma de tratamiento que tiene como objeto halagar al oyente para suavizar lo que ha dicho o va a decir posteriormente (Haverkate, 1994, p.88-117). Por tal razón, es común el empleo de sufijos diminutivos que atenúan el valor léxico por medio del cual se obtiene un beneficio por parte del emisor. En este caso, cuando se utiliza el cumplido como instrumento para conseguir un beneficio a favor del hablante mismo, este estaría ejerciendo su función más característica: persuadir. En el caso del apelativo, por ejemplo, es común el uso de amorcito, mientras que en el caso de los cumplidos, ojitos sería una forma muy común de uso del sufijo diminutivo -ito; dichos ejemplos ilustran usos pragmáticos del diminutivo que han sido poco valorados y estudiados en el español de Costa Rica. 


\section{Metodología}

Para iniciar, como se ha mencionado, nuestro estudio se basa en un corpus totalmente natural, tomado de una red social, lo que lo hace completamente auténtico. Se trata, así, de un corpus monolingüe, ya que solo se basa en el idioma español de hablantes del Valle Central de Costa Rica. Además, se considera un corpus cerrado, ya que se estableció una fecha para su recolección, la cual va desde el primero de mayo hasta el treinta y uno del mismo mes. Por enfocarse en un aspecto de la lengua, se trata de un corpus especial, sincrónico y simple, ya que no se basa en etiquetas de orden sintáctico o gramatical. Así mismo, la tipología empleada es textual; como se ha mencionado, se basa en conversaciones espontáneas tomadas de una red social.

Segundo, enfocados en los informantes, se estableció primero analizar solo a personas de un área geográfica específica de nuestro país: Heredia. Se pensó en descartar a personas que no pertenecieran a dicha área. Luego, se decidió incluir a personas de diferentes zonas del Valle Central (Alajuela, San José y Cartago) para poder así tener un grupo más representativo. Dicha información debía de aparecer en sus cuentas de Facebook, de lo contrario eran descartadas.

Tercero, se estableció un rango de edad entre los quince y treinta y cinco años de edad. Si bien es cierto que la historia de los diminutivos es bastante remota, se consideró que la gran mayoría de los usuarios de Facebook son personas jóvenes y no tanto personas más adultas, esto por razones obvias de intereses, conocimientos computacionales y de internet. Así mismo, se debe establecer la situación actual, y esto se hace considerando las generaciones más recientes pues estas son las que acarrean consigo los cambios lingüísticos.

En cuarto lugar, no se trató de escoger a las personas de una forma previa, es decir, no se escogieron los informantes por lo que escribían en su Facebook, ya que esto haría subjetivo el trabajo y los resultados no serían tan válidos. Así, solamente se escogieron a las personas por relación o amistad entre sí y por las limitantes mencionadas anteriormente. Cada informante fue clasificado por separado, y su corpus almacenado de manera digital en archivos aparte. Los archivos han sido codificados con las iniciales de los informantes. Paralelamente a los archivos de los informantes, se encuentran los archivos en los cuales se ha incorporado la información de ellos conocido como Header. Así, si un informante, por ejemplo, se llama Carlos Manuel Martínez, entonces la información de este informante se refleja en el archivo individual como C-M-M y su respectivo Header como C-M-M-H. Todos los subcorpus son las conversaciones entre estas personas durante el mes de mayo. Se trata de un total de veintitrés usuarios de Facebook, de los cuales cinco son hombres y dieciocho son mujeres. Sin embargo, se debe de considerar que cada usuario tiene su grupo de amigos con los cuales estableció sus conversaciones, por lo que la proporción hombre mujer no es tal. Además, los diminutivos que se consideraron para dicho trabajo son los siguientes: -ito, -ita, -illo, -illa, -cito, -cita, -ico, así como sus realizaciones plurales.

Por último, se debe mencionar que para hacer que los resultados fueran más válidos y fidedignos, se utilizaron, como complemento a la revisión 
manual, las herramientas tecnológicas Antconc y Atlas-Ti. Dichas herramientas permitieron un mejor y más rápido análisis de los datos de donde se substrajeron, a la vez, los datos tanto cuantitativos como cualitativos respectivamente.

\section{Análisis cuantitativo de los datos}

Con base en los resultados obtenidos a partir de la herramienta Antconc y con el análisis de los dos tipos de diminutivos con sus respectivas variantes de género así como plural que, según las investigaciones son más frecuentes del español panhispánico, se obtuvieron los siguientes resultados. Para la variante diminutiva formada por el sufijo -ito, según los Clusters obtenidos, se da un total de ciento diecinueve tipos o palabras distintas, y un total de ciento treinta y seis muestras (Tokens) que contienen dicha forma, siendo el diminutivo de persona majito el más habitual con una frecuencia de tres (ver ilustración 1). Para su correspondiente forma femenina -ita, los resultados son mayores. Así, se logró obtener un total de ciento noventa y dos Types (tipos) distintos de entradas y un número total de Tokens ${ }^{3}$ (muestras) de seiscientas veintiséis.

\section{Ilustración 1}

\section{Sufijo -ito}

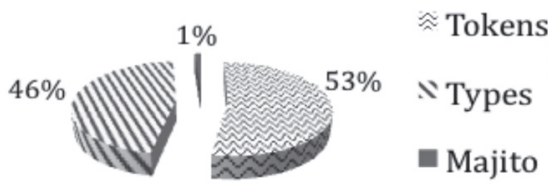

Para las formas sufijadas con el diminutivo -ito pero con referencia apelativa, la forma del sufijo -ito se ve reducida sustancialmente, siendo utilizada solamente en catorce muestras; entre dichas entradas léxicas figuran las formas gatitos, caracolitos y perritos. Para la primera forma se obtuvo un total de tres formas; para la segunda, un total de dos; y para la última, nueve muestras. Para otras palabras se dio en total un número de diecinueve tipos distintos (s/u 2), por lo que para este sufijo se da un número total de cuarenta y cinco tipos distintos de palabras. Al comparar los datos obtenidos en este estudio y las palabras mencionadas anteriormente con el corpus CREA, los resultados son sorprendentes ya que en este corpus solamente aparece la palabra gatitos en tres ocasiones (s/u 3).

\section{Ilustración 2}

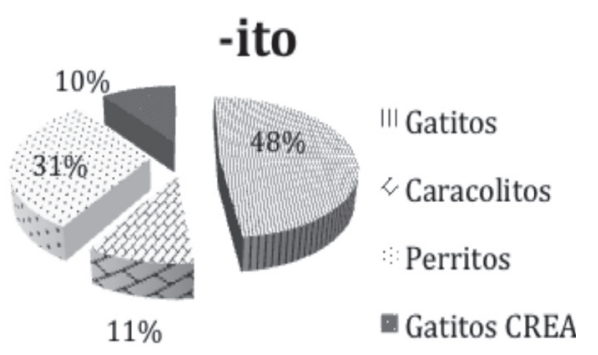

\section{Ilustración 3}

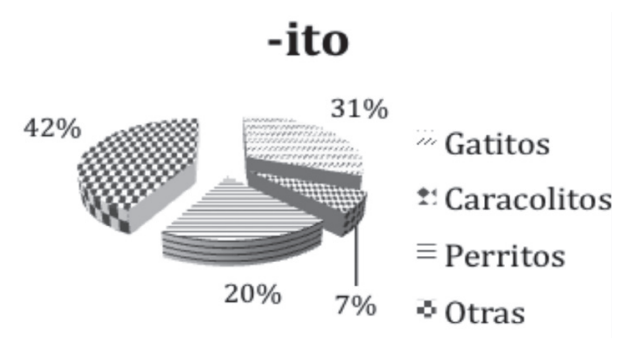




\section{Ilustración 4}

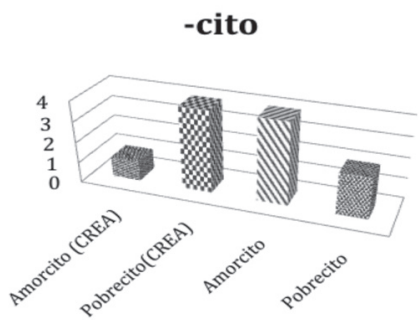

Para la forma -cito, se obtuvieron resultados un poco más elevados. Así, se localizó un total de doce Types y Tokens para esta forma, siendo amorcito, papacito y pobrecito las más comunes (s/u 4). Para la forma -illo, se detectó un total de cuatro tipos y cuatro Tokens, y para su forma -illa se detectaron tres formas, siendo brujilla y chiquilla las más dadas.

Si hacemos el mismo análisis utilizando el buscador de datos del corpus diacrónico CORDE, los resultados no son distintos, al obtener solo un resultado para pobrecito y ninguno para amorcito. Esto refleja, en cierta medida, la congruencia con los datos obtenidos en el presente estudio. Algunos de los sufijos diminutivos encontrados más frecuentemente en el corpus obtenido de Facebook son los siguientes:

\begin{tabular}{ccl}
\hline $\begin{array}{c}\text { Tipo de } \\
\text { diminutivo }\end{array}$ & Palabra & \multicolumn{1}{c}{ Contexto } \\
\hline & regalito & ...bien... ah y gracias por el regalito jajajaa! \\
caracolito & Ya no me gusta el caracolito egoísta agarro el alimento para el \\
& & solo... \\
& hermanito & Otro regalo de mi hermanito! \\
-ito & tarrito & $\ldots$...nada más que queda hacer el tarrito para la sepa de... \\
& amorcito & $\ldots$..feliz cn mi Amorcito ? ? ? \\
& perrito & $\ldots$ ESTE HERMOSO PERRITO BUSCA HOGAR \\
& papacito & $\ldots$...Papacito de mi corazón! Te amo muchísimo! \\
& tiempito & Tenga tiempito por vara! \\
& solito & ...vaga solito en el mundo y va deseando la muerte \\
\hline
\end{tabular}

Para las formas que contienen el sufijo diminutivo -ita, las formas más representativas son las siguientes:

\begin{tabular}{ccl}
\hline $\begin{array}{c}\text { Tipo de } \\
\text { diminutivo }\end{array}$ & Palabra & Contexto \\
\hline & adrianita & Feliz Cumpleaños Adrianita Bendiciones que \\
& flakita & Mi Flakita bella FELIZ CUMPLEAÑos \\
& papita & Karen y Papita Malcriada les extraño demaaaaaaa \\
-ita/-ito & bolsita & ...bolsita de azúcar y levadura cada mes casi 21 de mayo \\
& tiempito & El Tigre Sarita Poltronieri Tenga tiempito por vara \\
& viejita & ...viejita no los veo comer de arriba esperan a que les caiga... \\
& perrita & Hermosa perrita rescatada escondida entre matorrales...
\end{tabular}


casita $\quad$... busca casita...

vueltita ...apenas pueda me doy una vueltita $\mathrm{x}$ alla.

\section{Análisis pragmático}

De las formas más frecuentes incluidas en los diminutivos -ito/a, los resultados en términos de uso real son los siguientes. Para el primer caso, se dio un total de ciento diecinueve tipos o palabras distintas, y un total de ciento treinta y seis palabras; para el segundo caso, un total de ciento noventa y dos tipos distintos de entradas y un número total de seiscientas veintiséis muestras. Ambas estadísticas forman parte de sus formas singulares. Basados en un análisis tanto en Antconc como en Atlas-Ti, el cual contempla un análisis cualitativo de los datos, se pudo conocer que de las formas con -ito, un total de veintitrés palabras fueron utilizadas como formas afectivas. Por ejemplo, en yo AMO a mi bebito Prendiz muxo muxo. También, de estas formas, un total de dieciséis tipos para usos diminutivos como en es increíble lo rápido que pasa el tiempo, hace poquito estaba como en la foto y, por último, un total de cinco tipos para despectivos tales como Aaajajaja!! Pecaito con esa chiquilla!!. Cabe resaltar que se trata de tipos y no de muestras. En el caso del sufijo -ita y después del análisis con dichas herramientas, se obtuvo que veintidós tipos fueron utilizados como formas afectivas, mientras que un total de doce tipos distintos se utilizaron como diminutivos. Finalmente, solo seis tipos se emplearon como despectivos. Estas formas se aprecian y resumen en el siguiente cuadro donde se incluyen las más usuales extraídas de las conversaciones del corpus. (Cabe mencionar que acá se pretende resaltar la función pragmática del contexto como un contraste respecto de otros estudios y de posibles diferencias en los resultados desde la perspectiva semántica, en la cual se toma en consideración el contexto oracional).

\begin{tabular}{|c|c|c|}
\hline \multicolumn{3}{|c|}{ Diminutivos con -ito } \\
\hline & Contexto oracional & Uso del diminutivo \\
\hline 1. & es cansada esa actitud ante la vida y ODIO el pobrecito yo & Afectivo* \\
\hline 2. & nada mas que queda hacer el tarrito para la sepa de gusano de harina & Diminutivo \\
\hline 3. & feliz feliz cumple tito... muchas bendiciones.. & Afectivo \\
\hline 4. & solo uno mas y resistamos!!!!!!!, un golcito mas por fa!!!! & Diminutivo * \\
\hline 5. & Nadie me quiere todos me odian mejor me como un gusanito & Diminutivo \\
\hline 6. & yo te envío un mensajito mañana, confirmandote? & Diminutivo * \\
\hline 7. & woooooo se le ve azuladito el cachete sera macho deberas entonces!!! & Diminutivo* \\
\hline 8. & espero ganarme el regalito de cumpleaños jajaja & Afectivo \\
\hline & jajajaja q risa este perrito ! me da risa q diga lucho & Afectivo \\
\hline & $\begin{array}{l}\text { ESTE HERMOSO PERRITO BUSCA HOGAR, SE ENTREGA } \\
\text { VACUNADO, DESPARACITADO }\end{array}$ & Afectivo* \\
\hline & om nom nom!!! quequito!! para celebrar mi cumpleaños? & Diminutivo* \\
\hline
\end{tabular}


12. se acabo esto yaaaaaa por un ratito al menos!!!!uuuuhhhh. .

13. ...va cabalgando un jinete, vaga solito en el mundo y va deseando la muerte...

14. es increíble lo rápido que pasa el tiempo, hace poquito estaba como en la foto

15. Pro graziiaz priimiito lo kiiero un monton... :)

16. no ves que cosita mas HERMOSAAAA!!! Juntara este perrito 500 me gusta??

17. Papacito de mi corazón! Te amo muchísimo!

18. Aaajajaja!! Pecaito con esa chiquilla!!

19. Apoyemos a Pablito!! Apoyemos el arte puro y de verdad

20. mi hermana y mi novia...fijo no hablan de este morenito ;)
Diminutivo*

Afectivo

Diminutivo

Afectivo

Afectivo*

Afectivo

Despectivo

Afectivo

Afectivo
Al observar los datos del cuadro, se puede concluir en la columna de la derecha la función del diminutivo. No obstante, se puede observar que hay conclusiones marcadas por un asterisco $(*)$, el cual indica que la función o fuerza ilocutiva del diminutivo no está del todo clara según el contexto oracional. Esto indica que dichas formas deben de ser consideradas por separado en un contexto más amplio, como el lenguaje en uso, y no limitarse solo al oracional, el cual sería un análisis más semántico. Analizaremos el caso número 1 como ejemplo; para ello incluimos la conversación completa tomada como se dio en Facebook. Solamente modificamos los nombres de los hablantes incluyendo solo las iniciales:

MR: jaja yo ya mande hace meses la preinscripcion pero si vamos!! :D

NB: muy temprano!, pero puedo hacer el esfuerzo.. jajajaja

SCV: jajajajaja Valerii y Kenneth tienen $q$ ver esto!!!! esto si es un respeto de película no como aquella mier...q sacamos la vez pasada!!! esta si se ve BUENAA!! la tenemos $q$ ver oyeron!!! Jajajajaja

$S C V$ : jajajajaja buenisimooo!!cuidado xq después el mae le sale armado con la cuchara!!
VB: jaja oh rebe!!

$V B$ : No soporto la gente depresiva....

NB: no se meta conmigo!... jajajaja mentira XMMM: Ay si... De vez en cuando pasa pero hay unos ke todos los dias y si no es una cosa es otra... Uno se siente como en un equipo de porrismo $p$ ke la otra persona viva trankila ... Realmente es cansada esa actitud ante la vida y ODIO el pobrecito yo AJM: si, que fea la gente así!

Como se puede ver, por medio de un análisis pragmático, en el cual se toma en cuenta el contexto donde se da el acto ilocutivo en la conversación, el uso del diminutivo en el pobrecito yo no es afectivo como se podía suponer mediante el análisis aislado oracional y más bien constituye un uso despectivo. Quizás por la misma informalidad del género que se está analizando, se dan malas interpretaciones si se analizan en el nivel oracional, ya que el uso de comillas (" ”) podría haber esclarecido la intención real del hablante en esta conversación.

En el caso del segundo término con un asterisco (número 4), igualmente pareciera que a simple vista se trata de un diminutivo por lo que implica el contexto oracional, dado principalmente por el adjetivo un. Analizando 
el contexto donde este ocurre, podemos observar lo siguiente:

CAPM: vamos heredia podemos!!!! solo uno mas y resistamos!!!!!!!, un golcito mas por fa!!!!

CAPM: PARIDISIMOSSSSSSS!!!!!!! NOOOOOOO, NOOOOO

IE: JAJA .. ay amoor

CAPM: estoy que me como solo por dentro, ya no aguanto estar perdiendo

IE: haha .. Este este Emmss u.u JAJA? ay amoor (: (: RELAX

CAPM: toooomeeeeeeeennnnnnnnnnn putasssss!!!!!!!!, que lindo gol de team!!!

Al analizar la conversación en sí, se puede notar que el sustantivo que contiene el diminutivo -ito (golcito) claramente disminuye el valor del gol y lo hace ver hasta como algo insignificante que solamente es uno. Sin embargo, desde la perspectiva de la pragmática, dicho acto ilocutivo se da más bien con la clara intención afectiva del hablante, donde los demás elementos que componen el contexto hacen ver que el golcito es ni más ni menos que una gran necesidad para conseguir el objetivo: ganar. Así también, el mismo hablante lo expresa en los demás elementos dentro del contexto: estoy que me como solo por dentro expresa explícitamente que hay ansias de un gol que este hablante indica por medio de un sufijo -ito, dándole así a su proposición un acto ilocutivo de ansiedad y afecto reflejados por medio de este sufijo. También, se puede ver que hay un acto perlocutivo que el hablante busca: empatía. Sin embargo, como se mencionó anteriormente, dichos actos no siempre se consiguen $\mathrm{y}$, en este caso específico, el hablante solo obtiene de su interlocutor un acto perlocutivo de consuelo, expresado por la frase tomada del inglés relax. Veamos ahora el caso número 6:

IH: te extraño tanto

MFSB: bueno te amo muchooo pequee?, yo te envío un mensajito mañana, confirmandote? :')

IH: yo mas preciosa! xfa:)

MFSB: Y me envenenan los besos que voy dando $y$, sin embargo, cuando duermo sin ti contigo sueño, y con todas si duermes a mi lado.

Una vez más, similar al caso anterior, la palabra mensajito por sí sola, sin tomar en cuenta el contexto, denota una forma diminutiva. Pragmáticamente hablando, considerando los demás elementos del contexto, se puede ver que el hablante inserta muchos cumplidos alrededor del sustantivo mensajito; esto indica que el uso del sufijo es puramente afectivo por el contexto donde se da más que por el sufijo diminutivo empleado.

Veamos ahora el caso número 8 en el cual, desde una perspectiva semántica, el diminutivo es claramente afectivo. Comprobémoslo a partir del contexto de la conversación en sí:

VB: Chiquillas!! Gala Boutique está regalando 3 Órdenes de Compra por 100 Dólares solo tienen que hacerse Fan de su página, yo ya soy Fan :D espero ganarme el regalito de cumpleaños jajaja

SCV: noo!!

$S C V:$ jajajaja

VB: fea!

VB: Kenneth la doñita toda emocionada!!! jajaja

El caso número 10 de la tabla anterior parece ser aún más interesante que los vistos hasta este momento. Veamos el contexto: 
DP: Somos una asociación sin fines de lucro que ayuda a los animalitos de la calle, los recursos que tenemos son pocos y los gastos muchos, actualmente tenemos muchos casos de perritos y gatitos y cada día se nos presentan más, necesitamos de ...

DP: ESTE HERMOSO PERRITO BUSCA HOGAR , SE ENTREGA VACUNADO, DESPARACITADO Y CASTRADO . MAS INFORMACIÓN LE BRINDAMOS POR MSJ PRIVADO

DP: jajaja asi o mas feo jajaja

Sin duda alguna, si se nos presenta solo el contexto inicial, donde el hablante inicia aclarando que son una asociación sin fines de lucro y demás, y luego, este mismo informante hace el anuncio donde expresa que es un lindo perrito, diríamos que se trata claramente de un sufijo -ito agregado a un sustantivo perro con un contenido proposicional "hermoso + perro+ buscar+ hogar", el cual denota un acto perlocutivo de piedad y apoyo. Sin embargo, más adelante el contexto nos aclara que el hablante no hace más que un acto ilocutivo (que desencadena en un acto perlocutivo de burla) por medio de dicha proposición al indicar jajaja asi o mas feo jajaja. Queda así reflejada la importancia de considerar el contexto a la hora de analizar los actos de habla de los hablantes y las locuciones que ellos hacen. En fin, se trata no de un sufijo diminutivo con valor afectivo sino más bien despectivo. Similarmente, si nos limitamos a lo que los signos lingüísticos implican en la oración número 11, vemos que quequito no es más que la reducción de queque por medio del diminutivo -ito. No obstante, observando la conversación donde se da dicho "diminutivo", podemos deducir que se trata de un diminutivo con valor afectivo:
KA: jaja no no a mi solo me gustan los bares no los clubes jaja

VB: Trauma infantil al chico que se lo compren. $x D$

SCV: noooooooo!!! jajaja le comprare uno val!!

VB: awwww yo seria muy feliz!! jaja VB: om nom nom!!! quequito!! para celebrar mi cumpleaños?

Para los demás casos marcados con (*), parece que el contexto donde se dan es un poco oscuro y no está del todo claro el uso real del sufijo por lo que sería arriesgado dar una conclusión definitiva.

Veamos ahora algunos ejemplos de las formas construidas por el sufijo -ita en un contexto más amplio que el oracional. Empecemos con un claro ejemplo donde el objeto ilocutivo del acto de habla expresado por medio de este sufijo es distinto del que se espera, es decir, no se busca disminuir el valor de la palabra a la que se le ha agregado dicha forma.

VB: Chiquillas!! Gala Boutique está regalando 3 Órdenes de Compra por 100 Dólares solo tienen que hacerse Fan de su página, yo ya soy Fan :D espero ganarme el regalito de cumpleaños jajaja

$S C V:$ noo!!

$S C V:$ jajajaja

VB: fea!

VB: Kenneth la doñita toda emocionada!!! Jajaja

En esta conversación se da, claramente, el empleo del elemento -ita para expresar por medio de este acto ilocutivo una forma despectiva añadida a la forma léxica doña. Algo similar sucede en la siguiente conversación, donde se está comentando un producto que aparece en una foto. 
CAPM: vea esta mae, super sencillita $y$ ronda los 3 mil dolares, y no tiene asi mucha mierdita, ni la marca esta en el marco parece paquetasa

LP: Se la voy a pedir al niño...!!!!!

Primero, podemos ver que el hablante CAPM utiliza el sufijo como modo de disminuir el valor del artículo, añadido a una palabra que por sí sola ya contiene un valor negativo, haciéndolo de esta manera aún más negativo, por lo que el valor despectivo o negativo del artículo aumenta. Asimismo, podemos notar que el hablante trata de conseguir (acto ilocutivo) un apoyo a su punto de vista (acto perlocutivo). LP, por medio de su expresión, parece estar de acuerdo con dicho punto de vista, al apoyar la imagen positiva de CAPM. Además, parece que en la segunda palabra subrayada mierdita se hace un uso similar al anterior; no obstante, podemos agregar algo más: se trata de mitigar por medio del sufijo -ita la aserción o argumento que CAPM hace sobre este artículo, que si bien es cierto que LP acepta como tal, CAPM desconocía cuál iba a ser el punto de vista de LP, por lo que la mitigación fue una buena forma de cuidar la imagen de LP (así como la de él mismo), ya que dicha proposición podía ser un acto fallido. En la siguiente conversación, podemos ver más claramente cómo se trata de mitigar el acto de habla y cómo se trata de cuidar, por medio del sufijo, la imagen positiva del interlocutor:

VB: mae... por que putas el semestre no se acaba YA??!!

VB: jaja mañana voy y saco el permiso $p$ andar una d esas!!! jajaja

SCV: jajaja Exelenteee
RS: haha. les recuerda $\mathrm{cm}$ un estival d talentos viejo??

SCV: jaja siiiii q belleza!!! Rebeca mi compañera de baile te acordás???

VB: SS vease!! jajaja

DLC: jajajajajajajajajajajajaja peroooo igualita!!!!!

En esta conversación, se está comparando a uno de los hablantes con alguien que aparece en una foto. DLC trata de mitigar su acto ilocutivo utilizando la forma igualita, la cual a su vez cuida la imagen positiva de SS. Veamos el siguiente caso:

DFR: QUE VERGUENZAAA...

LS: Ishh q programita tan apestoso! QUEREMOS UNA SOCIEDAD MÁS INCLUSIVA, NO ACEPTAR ÉSTO ES HOMOFOBIA KHA: no no pero simplemente es para dar un ejemplo extremo de tolerancia jajajaj DFR: jajajajajajaja ah ok ya quedo claroo..

DFR da un juicio personal sobre el programa que KHA etiquetó. LS también da su opinión y KHA lo hace nuevamente. Como es claro, KHA trata de conservar y proyectar su imagen. Primero, trató de ser aceptado y que su imagen positiva fuera reconocida mediante la publicación hecha. Contrariamente, LS, por medio del sufijo -ita, daña la imagen positiva de KHA y este reacciona a dicho rechazo. Al no aprobar el video, se da un Face-Theatening Act, por lo que se mitiga el acto por medio del diminutivo con valor despectivo. En resumen, el acto ilocutivo de KHA no consigue la perlocución deseada, lo que afectó su imagen positiva. Parece ser que LS sí consiguió la perlocución deseada por medio de su acto ilocutivo: desvalorizar la opinión de KHA. Veamos ahora un caso contrario: 
AAC: Bueno chic@s: ahora lo que sigue es la incorporación, ojalá y todos nos incorporemos en la misma fecha. Les adjunto el link para que tomen nota: http://www.cpcecr. com/incorporaciones.php - con Evelyn Arroyo y 3 personas más.

GMC: Siiiiiiiiiiiiiiii jajaja [nombre de la persona] siempre adelantadita con la info... yo la iba a buscar en estos días jajajaja. :D

En este caso, AAC hace una contribución y GMC claramente refuerza la imagen positiva de AAC por medio de su comentario y principalmente mediante el sufijo contenido en la palabra adelantadita. Así entonces, AAC consigue su acto perlocutivo convencional: un halago a su imagen positiva. Veamos este caso, el cual es muy similar:

SL: eres todo eso y MÁSH, mi gordito!! Te Amo Demashiadoooooooo!!!!! ? ? ? sos un BELLO!! TE AMOOO!!!

JC: Gracias manita por ser ese Angel que necesitaba, gracias por ser esa persona especial que no solo es mi amiga, sino un gran instrumento que Dios envio a mi vida para recordar lo mucho que nos ama y lo maravilloso que es...

SL: Sos una bella... y yo recibo con honor esa bendición y la comparto con tigo!! :*

Primero, SL hace uso del cumplido (bello, por ejemplo) así como por medio del sufijo -ito (gordito) para reforzar la imagen positiva de su interlocutor. Este último, a su vez, refuerza la imagen de SL haciendo uso de la misma estrategia conversacional: el cumplido (Ángel) y el sufijo afectivo -ita. Acá, entonces, se puede notar que ambos hablantes consiguen el acto perlocutivo de sus actos ilocutivos, lo cual está dado por la relación de cercanía (distancia social) de ambos hablantes y el rol que desempeñan en la sociedad, donde ambos cooperan y mantienen un balance. Como ilustración podemos considerar el siguiente ejemplo:

CAPE: Desearle por este medio a todas las madres de mi bello país, un feliz día de la madre, que Dios les haya regalado un grandioso día. En especial a mi madre GEB, que la amo mucho!!!

AFA: Dios bendiga a tu mamita $x$ haberte traido al mundo...? :-*?

AFA hace uso del cumplido y cuida, a su vez, la imagen positiva de un tercero (la mamá) que claramente juega un papel de cercanía social con CAPE; es decir, al cuidar la imagen positiva de esta persona está cuidando la imagen positiva de CAPE.

Cuando se analizan las formas terminadas en -illo/-illa, las cuales como se mencionó anteriormente son sufijos que dieron base a formas referenciales, se pudo constatar lo siguiente. En el caso de -illo, es frecuente encontrarlo en apellidos o nombres propios ${ }^{4}$. Así, Murrillo es bastante común en el corpus o bien Ocontrillo, lo cual representa un problema a la hora de contabilizar las formas que contienen dicho sufijo. Para este sufijo, es más común observar formas como atrasadillo, pepillo, carajillo, ritmillo, poquillo, o ratillo, las cuales contemplan un sentido más despectivo. Por ejemplo en Que carajillo más malcriado se puede observar cómo se emplea la forma -illo con un fin negativo. Para esta forma se encontró un total de 52 muestras y 41 tipos, lo cual la hace bastante frecuente. Para la forma -illa sucede algo similar. Apellidos como Bonilla y Chinchilla son frecuentes. Para este sufijo se dio un total de 49 muestras y 38 tipos. 


\section{Ilustración 5}

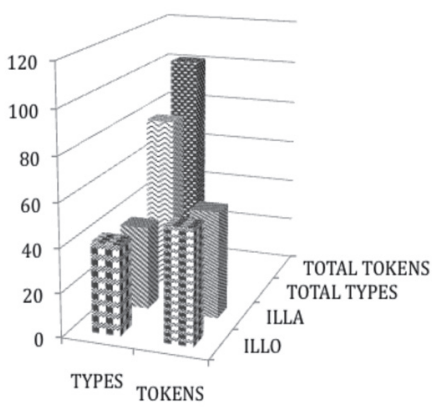

Algunos otros ejemplos no están tan claros si se analizan desde su contexto oracional, por lo que una vez más se debió contemplar el contexto completo donde estas formas se dan:

SO: Feliz cumple atrasadillo... que Dios me la bendiga mucho y espero que la hayas pasado del exito bella mil besos

AV: Atrasadillo pero bienvenido!! :) gracias Sill, TQM, un abrazo!

Parece ser que SO trata de cuidar su imagen positiva, empleando el sufijo -illo, el cual a su vez funciona como diminutivo: "solamente me atrasé un poco con la felicitación del cumpleaños." AV, por su parte, emplea una repetición del mismo sufijo y la misma palabra pero parece ser, por el contexto, que este hablante también minimiza el evento. Quizás por la conjunción pero podríamos suponer que se hace un uso más despectivo del sufijo que contrastaría con el uso empleado por SO. Recordemos que las ideas que se unen por conjunciones de contradicción como pero usualmente implican que una es positiva y la otra negativa. Por ser la expresión bienvenido positiva, podemos suponer que atrasadillo conlleva un significado más negativo; además, el uso de cumplidos posteriores a dicha ex- presión $(T Q M)$ parecen ser estrategias de atenuación para mitigar el efecto causado por el uso despectivo del sufijo -illo. Otros ejemplos son más evidentes:

SH: Que carajillo mas malcriado... no viste lo que pase ayer... y eso me pasa por decirle que solo usted me levanta el animo, algo asi como Patch Adams....

CAPM: woooooo el es un lindo(todo gay) esa peli la ameee

Claramente se hace el empleo de un sufijo -illo para denotar un acto despectivo que atenta claramente contra la imagen positiva del oyente. CAPM, por su parte, parece evadir dicho acto y se enfoca en la valoración de su propia imagen positiva, la cual fue elevada por SH en el comentario anterior. Así, SH parece no conseguir su acto perlocutivo principal (buscar empatía que apoyara el daño hecho a la imagen positiva de aquel referido por la expresión carajillo). Similarmente, CAPM hace un uso despectivo claro por medio de dicho sufijo en el siguiente comentario:

CAPM: compartió un enlace.

no se por que pero me gusta, tiene ritmillo vacilon!!

En el caso del sufijo -illa, podemos notar usos, aparte de la RAE y de los despectivos, distintos de los comunes:

CAB: Feliz cumpleaños brujilla, que Dios te bendiga y te regale un chorro de salud.

DDE: FELIZ CUMPLE :)

KGC: Gracias!! Muchas gracias a todos los que se acordaron de mi cumple

Sin un contexto, la palabra brujilla contemplaría un acto claramente despectivo; no obstante, considerando 
dicha palabra desde un enfoque más pragmático, tomando en cuenta el contexto donde ocurre, podemos notar que se trata de un empleo más afectivo. Por medio del contexto se puede ver cómo, fuera de lo común, se refuerza la imagen positiva del interlocutor por medio de una palabra que implica, normalmente y semánticamente (por su significado convencional), una idea no muy positiva (las brujas son normalmente malas). Dicho significado convencional (aquel que es dado por el diccionario) es atenuado por medio del sufijo y utilizado para significar algo positivo que funciona como apelativo, reforzando así la imagen positiva de KGC. Esta última, por su parte, está consciente de tal hecho y expresa explícitamente que el acto ilocutivo de sus interlocutores ha sido aceptado. Se completa así el acto por medio de la perlocución: felicitar al receptor.

Enfocados en las formas con -ico/$i c a$, las cuales se supone que dieron origen a la designación actual de $t i c o^{5}$, debemos mencionar que los resultados fueron sorprendentes. Primero que todo, para la forma -ico no se dan resultados algunos; es decir, parece que los informantes seleccionados no están haciendo uso de dichas formas que, se supone, identifican a la población costarricense. Para la forma -ica, solamente se da una entrada léxica conformada por la palabra chikitica ${ }^{6}$ (escrita literalmente del corpus). A continuación, ilustramos el contexto donde se utilizó dicha forma:

SCV: es q uds tomaron la foto y yo no sabia q habia q hacer tierno!! ves!! jajaja

EG: ?.l. Jajaja mae esq la roja ya estaba borracha!!

RLF: jaja elin vea la palma bendita q puso meri jou atras!! jaja pecao es tan chikitica q ni salio solo las manos con unas ramas. EG: Aaajajaja!! Pecaito con esa chiquilla!!

Sin duda alguna, esta forma hace referencia a un valor despectivo y negativo, donde es utilizada, más que para disminuir el valor léxico de la palabra, para afectar la imagen positiva de la persona a la cual es referida. Se trata así de un acto meramente descortés por parte de los hablantes donde se utilizan formas despectivas para burlarse (mediante un ataque a la imagen positiva) del interlocutor que está siendo afectado.

\section{Conclusiones}

Considerando los objetivos planteados desde el inicio de la investigación, podríamos decir que, al menos en el grupo investigado, la forma diminutiva - $(t) i c o / a$ presenta una de las frecuencias más bajas del estudio, por lo que el gentilicio parece no ser una forma originada del uso excesivo de los diminutivos en la población costarricense. Hipotéticamente, podríamos decir que dichas formas -(t)ico/a pudieron ser más frecuentes en el pasado que en la actualidad o, al menos, por los jóvenes de hoy. Sin embargo, para comprobar dicha hipótesis se debe realizar un estudio más amplio en diferentes áreas del país y tomar en cuenta una población de edad mayor. Por el momento, y basados en los resultados de esta pequeña población, la forma del diminutivo no parece ser la responsable del gentilicio.

Según las teorías utilizadas para este trabajo, las cuales abarcan mayormente diminutivos del español de países distintos de Costa Rica, con 
excepción de algunos artículos, la gran mayoría considera que el diminutivo más utilizado es -ito. Así lo recalcan Sien y otros autores mencionados previamente. Lo más resaltante de este trabajo, y quizás lo más inesperado, es que el diminutivo que distingue a nuestra población costarricense, al menos en este pequeño corpus, no es utilizado. Se esperaría que palabras como chiquitico fueran más frecuentes; sin embargo, esta palabra solo aparece una vez en jaja pecao es tan chikitica $q$ ni salió solo las manos con unas ramas. Lo mismo sucede con muchas otras palabras que se supone utilizan más las formas - (t)ico pero que, no obstante, los informantes no están haciendo uso de ellas, y utilizan más bien los diminutivos comunes como -ito/ -ita. Por tal razón, se puede decir que en la actualidad las formas diminutivas de Costa Rica no son para nada desiguales de las encontradas en otras regiones como El Salvador o España, donde -ito/a vienen a ser las dos formas más utilizadas. Incuestionablemente, se pudo comprobar que dicho sufijo acompañado de su forma femenina -ita son los más utilizados por los informantes que constituyeron el corpus base para la elaboración de este estudio. Así mismo, se pudo constatar la teoría de Zúñiga, ya que como bien lo apunta en su investigación, las formas -ito/-ita son mayormente utilizadas para referirse a situaciones de orden afectivo. La gran mayoría de los informantes de este estudio así lo hacen ver. Asimismo, las formas compuestas por el sufijo -illo/-illa son mayormente empleadas con fines despectivos. Dicha estadística también se pudo comprobar tanto desde la perspectiva semántica como pragmática.
No obstante, cabe señalar que por medio de la diferenciación semánticopragmática se pudieron obtener resultados diversos. Desde la perspectiva de la semántica, los resultados hubieran sido un tanto erróneos ya que, como se mostró con los resultados y a través de las ilustraciones, muchas de las formas que, basadas en el contexto inmediato oracional (co-texto), parecían ser diminutivas o bien afectivas, resultaban ser de otra índole. Por tal motivo, otra conclusión importante de este trabajo debe recaer en la importancia del contexto como una forma de establecer relaciones reales de significado entre los enunciados e ilocuciones hechos por los hablantes así como los actos perlocutivos que estos buscan a partir de los sufijos diminutivos. Dicho contexto condicionó la aparición de diminutivos usados como tales o bien con una doble función, donde una misma forma se utilizó como afectiva en unos casos y diminutiva en otros, o bien despectivamente. Además, se debe señalar la multifuncionalidad de estos sufijos en el español de Costa Rica, los cuales mantienen su función en el acto ilocutivo de disminuir el valor de la entrada léxica, hasta funciones de habla en sí. Así, formas afectivas, diminutivas, despectivas, apelativos, formas de cortesía como la atenuación de aserciones y cumplidos, entre otras, son asignadas a estos sufijos.

Se pudo notar, entonces, que la mayoría de los diminutivos del español de los informantes bajo estudio son formas para denotar afecto; es decir, diminutivos con valor afectivo. Formas como hermanito, perrito, Majito y amorcito, entre muchas otras, denotan no una forma de disminución sino, más bien, una forma de expresar afecto al 
interlocutor. Como forma de ejemplo podemos ver que en Apenas para mi amorcito Carlos Andrés, el diminutivo utilizado en amorcito denota, sin discusión alguna, una forma de afecto hacia la persona a la cual va dirigido el mensaje. Si bien es cierto que el sustantivo amor ya por sí solo expresa afecto, el uso del diminutivo le da un valor extra y, en vez de disminuir su valor semántico, más bien lo aumenta. Otro ejemplo con una palabra menos evidente es Otro regalo de mi hermanito!... siempre vas a hacer como mi hermanito. Claramente, la palabra hermano no denota por sí misma un afecto, el cual es agregado por -ito al final. Así se puede obtener infinidad de ejemplos como yo AMO a mi bebito Prendiz muxo тuxo muxooooooo :-* ? !!!! Carlos Andrés, y los mencionados anteriormente que evidencian una forma del diminutivo ito/a que expresa afecto. En resumen, se pudo constatar que las formas más recurrentes de los sufijos diminutivos se utilizan para demostrar afecto, seguido de disminución $\mathrm{y}$, finalmente, con valor despectivo. En comparación con los artículos e investigaciones hechas en el área centroamericana así como en España, la forma para demostrar afecto es la que más concuerda con los diferentes estudios. Las demás formas no han sido del todo estudiadas internacionalmente, por lo que hacer una comparación sería irresponsable.

Finalmente, no se puede dejar de lado el valor inherente del diminutivo, el cual en este estudio sucede con frecuencia por medio de -ito/a. Formas como se le ve azuladito el cachete será macho deberas entonces demuestran el valor diminutivo. Algunos otros ejemplos son nada más que queda hacer el tarrito para la sepa de gusano de hari- na o yo te envío un mensajito mañana, confirmándote. Por otro lado, formas despectivas y aquellas que son utilizadas para expresar lástima o emotividad son también bastante frecuentes. En la oración ESTE HERMOSO PERRITO BUSCA HOGAR, SE ENTREGA VACUNADO, DESPARACITADO, claramente se nota el uso del diminutivo como una forma de expresar lástima, empleado de forma consciente para conmover al informado. Aún más clara es la forma pecaito con esa chiquilla!!, donde evidentemente se expresa un valor de afecto, lá 7 stima e inferioridad hacia la persona utilizando el diminutivo.

\section{Notas}

1 https://es-es.facebook.com/legal/ terms?locale=es_ES

2 Una forma de tratamiento hace referencia a ciertas palabras, como pronombres, utilizadas por los hablantes para referirse, con cierta intención, a sus interlocutores con el fin de lograr un propósito (sea positivo o negativo para alguna de las partes).

3 Types/Tokens son términos utilizados por la herramienta Antconc, por lo que se decidió respetar dichos nombres y colocar sus posibles contrapartes en español.

4 Cuando se menciona el caso de los apellidos o nombres propios como $\mathrm{Mu}$ rillo/Ocontrillo, no se está implicando ni se debe entender que son los mismos usuarios que se autodefinen de esta forma sino, más bien, se da el caso en que las personas utilizan los apellidos como formas apelativas para referirse a sus amigos/as.

5 Gentilicio equivalente a costarricense.

6 No está de más mencionar que la forma diminutiva para la palabra chiquita sería 
chiquitita; no obstante, en Costa Rica, se da un proceso de disimilación, en este caso de /c/, resultando en chiquitica ( ${ }^{*}$ chiquititica o chirrisquitica), razón por la cual surgió el gentilicio mencionado anteriormente.

7 La bibliografía ofrecida se refiere solamente a la que fue citada en este artículo.

\section{Bibliografía $^{7}$}

Borja, L. (2010). Español salvadoreño. Uso del diminutivo en el habla de comerciantes de Santa Ana. <http:// espanolsalvadoreno.blogspot. com/2010/11/uso-del-diminutivoen-el-habla-de-los.html>

Brown, P. y Levinson, S. (1987). Politeness: Some universals in language usage. Cambridge: Cambridge University Press. [First published 1978 as part of Ester $\mathrm{N}$. Goody (ed.): Questions and Politeness.]
Callebaut, S. (2010-2011). Entre sistematización y variación: El sufijo diminutivo en España y en Hispanoamérica. Universiteit Gent.

Haverkate, H. (1994). La cortesía verbal: estudio pragmalingüístico. Madrid: Gredos.

Hummel, M. Para la lingüística de Vuestro Diminutivo. Universidad de Marburg.

Searle, J.R. (1980). Actos de habla. Madrid: Cátedra.

Spitzer, L.(1921). Reseña crítica del primer estudio de Amado Alonso. $<<$ Das Suffix -one im Romanischen $>$, en E. Gamillscheg, L. Spitzer, Beitráge zur romanischen Wortbildungslehre, Genf.

Vidal, E. (1996). Introducción a la pragmática. Barcelona: Editorial Ariel. 